\title{
NOTES ON GRAPHS WITH LEAST EIGENVALUE AT LEAST -2*
}

\author{
JIANFENG WANG $^{\dagger}$, YUFA SHEN ${ }^{\ddagger}$, AND QIONGXIANG HUANG ${ }^{\S}$
}

\begin{abstract}
A new proof concerning the determinant of the adjacency matrix of the line graph of a tree is presented and an invariant for line graphs, introduced by Cvetković and Lepović, with least eigenvalue at least -2 is revisited and given a new equivalent definition [D. Cvetković and M. Lepović. Cospectral graphs with least eigenvalue at least -2. Publ. Inst. Math., Nouv. Sér., 78(92):51-63, 2005.]. Employing this invariant and other techniques, it is shown that the line graphs of double stars are determined by their adjacency spectra.
\end{abstract}

Key words. Adjacency matrix, Least eigenvalue -2 , Spectral characterization, Generalized line graph, Double star.

AMS subject classifications. 05C50.

1. Introduction. In this paper, we are concerned only with undirected simple graphs (loops and multiple edges are not allowed). All notions on graphs that are used here can be found in [5]. For a graph $G=(V(G), E(G))$, let $n(G), m(G), \ell(G)$ and $A=A(G)$ be the order, size, line graph and adjacency matrix of $G$, respectively. For some vertex $v_{i} \in V(G)$, let $d_{i}=d\left(v_{i}\right)$ stand for the degree of $v_{i}$ and $\Delta(G)$ be the maximum degree of $G$. We denote the characteristic polynomial $\operatorname{det}(\lambda I-A)$ of $G$ by $\phi(G, \lambda)$ or simply $\phi(G)$. The adjacency spectrum of $G$, denoted by $\operatorname{Spec}(G)$, is the multiset of eigenvalues of $A(G)$. Since $A(G)$ is symmetric, its eigenvalues are real and we set $\lambda_{1}(G) \geq \lambda_{2}(G) \geq \cdots \geq \lambda_{n(G)}(G)$. The maximum eigenvalue $\lambda_{1}(G)$ of $G$ is called the spectral radius (or index) of $G$ and it is often denoted by $\rho(G)$. Additionally, let $\lambda(G)$ denote the least eigenvalue of $G$.

Two graphs $G$ and $H$ are said to be $A$-cospectral if the corresponding adjacency spectra are the same. A graph is said to be determined by the A-spectrum (or simply a

\footnotetext{
* Received by the editors on September 13, 2011. Accepted for publication on March 24, 2012. Handling Editor: Bryan L. Shader.

${ }^{\dagger}$ Department of Mathematics, Qinghai Normal University, Xining, Qinghai 810008, P.R. China (jfwang4@yahoo.com.cn). Supported by National Natural Science Foundation of China for Young Scholar (no. 11101232) and National Natural Science Foundation of Qinghai Province for Young Scholar (no. 2011-Z-929Q).

${ }^{\ddagger}$ College of Mathematics and Information Science and Technology, Hebei Normal University of Science and Technology, Qinhuangdao, Hebei 066004, P.R. China (syf030514@163.com). Supported by the Mathematical Research from the Natural Science Foundation of Hebei Province, China (no. 08M004).

$\S$ College of Mathematics and System Science, Xinjiang University, Urumqi 830046, P.R. China (huangqx@xju.edu.cn). Supported by National Natural Science Foundation of China (no. 10961023).
} 
$D A S$-graph) if there is no non-isomorphic graph $A$-cospectral to it, i.e., $\phi(G)=\phi(H)$ implies $G \cong H$. The background of the question "which graphs are determined by their spectrum?" originates from chemistry (in 1956, Günthadr and Primas 16] raised this question in the context of Hückel's theory). For additional remarks on the topic we refer the readers to [11, 12]. There are many papers on $A$-cospectral graphs and the methods used to construct them (see [14, 17, 18, 20, for example). By contrast, the question of determining what kinds of graphs are DAS-graphs is far from resolved. See [1, 22, 23, 24, 28, 29, 30, 31] for some recent results in this field.

Some other notation and terminology are also needed. Let $G_{1} \cup G_{2}$ denote the disjoint union of graphs $G_{1}$ and $G_{2}$, and $k G_{1}$ the disjoint union of $k$ copies of $G_{1}$. Let $n_{G}(H)$ denote the number of the subgraphs $H$ in graph $G$. As usual, let $P_{n}, K_{n}$ and $K_{1, n-1}$ denote the path, the complete graph and the star of order $n$ respectively. Let $S_{a, b}$ be the double star obtained from the stars $K_{1, a}$ and $K_{1, b}$ by joining the vertex of degree $a$ in $K_{1, a}$ and the vertex of degree $b$ in $K_{1, b}$ (see Fig. 4.1). Note that $S_{0, b}=$ $K_{1, b+1}$. The well-known cocktail-party graph $C P(k)$ is obtained from $K_{2 k}$ by removing $k$ disjoint edges. Hoffman [19] introduced the generalized line graph as follows: for any graph $H$ with $n$ vertices $v_{1}, v_{2}, \ldots, v_{n}$ and any non-negative integers $a_{1}, a_{2}, \ldots, a_{n}$, then the generalized line graph $L\left(H ; a_{1}, a_{2}, \ldots, a_{n}\right)$ is the graph consisting of disjoint copies of $\ell(H)$ and $C P\left(a_{i}\right)$ together with additional edges joining a vertex in $\ell(H)$ with a vertex in $C P\left(a_{i}\right)$ if the vertex in $\ell(H)$ corresponding to an edge in $H$ has $v_{i}$ as an end-vertex $(i=1,2, \ldots, n)$. The following theorem is a well-known result relating to generalized line graphs.

Theorem 1.1. (Cameron, Goethals, Seidel, and Shult [3]) Let $G$ be a connected graph with least eigenvalue at least -2 . Then either $G$ is a generalized line graph or $G$ can be represented by vectors in the root system $E_{8}$.

Graphs with least eigenvalue at least -2 have been studied since the very beginnings of the theory of graph spectra. Much information on this field can be found in the books [2, 4, 9, 15].

It is an interesting problem to find which graphs with least eigenvalue at least -2 are $A$-cospectral graphs or DAS-graphs. Here we mention some known results. An exceptional graph is a connected graph with least eigenvalue at least -2 which is not a generalized line graph. Cvetković and Lepović [6, 7] studied the phenomenon of $A$-cospectrality in generalized line graphs and in exceptional graphs. For the regular DAS-graphs with least eigenvalue at least -2 , van Dam and Haemers [1] gave an almost complete answer (see their Theorem 8). Further results on $A$-cospectral graphs may be found in Section 4.2 of [9]. However, for the non-regular case, van Dam and Haemers [1] stated that the following question remains open.

Problem 1.2. Which non-regular graphs with least eigenvalue at least -2 are 


\section{DAS-graphs?}

This paper is organized as follows: In Section 2, we cite some useful results of graphs with least eigenvalue at least -2 , and give a new proof of an important result about the determinant of adjacent matrix of line graph of a tree. In Section 3, we revisit an invariant, defined by Cvetković and Lepović $[8$, for the graphs with least eigenvalue at least -2 , and give it a new equivalent definition. In Section 4 we prove that the line graphs of double stars are determined by their adjacency spectra.

2. Some useful results and a new proof. Doob and Cvetković [13] characterized all connected graphs with the least eigenvalue greater than -2 as follows:

THEOREM 2.1. $G$ is a connected graph with $\lambda(G)>-2$ if and only if one of the following holds:

(i) $G \in \mathcal{G}_{1}=\{\ell(T) \mid T$ is a tree $\}$;

(ii) $G \in \mathcal{G}_{2}=\{L(T ; 1,0, \ldots, 0) \mid T$ is a tree $\}$;

(iii) $G \in \mathcal{G}_{3}=\{\ell(H) \mid H$ is an odd-unicyclic graph $\}$;

(iv) $G \in \mathcal{G}_{4}=\left\{20\right.$ graphs with order 6 that are represented in $\left.E_{6}\right\}$;

(v) $G \in \mathcal{G}_{5}=\left\{110\right.$ graphs with order 7 that are represented in $\left.E_{7}\right\}$;

(vi) $G \in \mathcal{G}_{6}=\left\{443\right.$ graphs with order 8 that are represented in $\left.E_{8}\right\}$.

For convenience, set $\mathscr{L}=\{G \mid G$ is a connected graph and $\lambda(G) \geq-2\}, \mathscr{L}^{+}=$ $\{G \mid G$ is a connected graph and $\lambda(G)>-2\}$ and $\mathscr{L}^{0}=\{G \mid G$ is a connected graph and $\lambda(G)=-2\}$. Clearly, $\mathscr{L}=\mathscr{L}^{+} \cup \mathscr{L}^{0}$ and $\mathscr{L}^{+}=\mathcal{G}_{1} \cup \mathcal{G}_{2} \cup \mathcal{G}_{3} \cup \mathcal{G}_{4} \cup \mathcal{G}_{5} \cup \mathcal{G}_{6}$. A graph in $\mathscr{L}\left(\mathscr{L}^{+}\right.$or $\left.\mathscr{L}^{0}\right)$ is called an $\mathscr{L}$-graph $\left(\mathscr{L}^{+}\right.$-graph or $\mathscr{L}^{0}$-graph) (see [8]). For the $\mathscr{L}^{+}$-graphs we have the following result.

Theorem 2.2. (Brouwer, Cohen, and Neumaier [2]) Let $G$ be an $\mathscr{L}^{+}$-graph with order $n$. Then

$$
\operatorname{det}(2 I+A(G))=\left\{\begin{array}{cl}
n+1 & \text { if } G \in \mathcal{G}_{1} ; \\
4 & \text { if } G \in \mathcal{G}_{2} \cup \mathcal{G}_{3} ; \\
3 & \text { if } G \in \mathcal{G}_{4} ; \\
2 & \text { if } G \in \mathcal{G}_{5} ; \\
1 & \text { if } G \in \mathcal{G}_{6} .
\end{array}\right.
$$

The above theorem has been proved by a lattice-theoretic argument (see, for example, 2]). It is worth pointing out that Doob and Cvetković [13] gave a proof for the case $G \in \mathcal{G}_{2} \cup \mathcal{G}_{3}$ by other techniques. Here, we also give a new proof for the case $G \in \mathcal{G}_{1}$ which is independent of lattice theory. Let $\psi(G, \lambda)=\operatorname{det}(\lambda I-(D-A))$ and $\varphi(G, \lambda)=\operatorname{det}(\lambda I-(D+A))$ be respectively the characteristic polynomials of 
Laplacian matrix $D-A$ and signless Laplacian matrix $D+A$ of $G$, where $D=D(G)=$ $\operatorname{diag}\left(d_{1}, d_{2}, \ldots, d_{n(G)}\right)$ is the degree matrix of $G$.

LEMma 2.3. 10] Let $G$ be a graph with order $n$ and size $m$. Then

(i) $\varphi(G, \lambda)=\lambda^{n-m} \phi(\ell(G), \lambda-2)$.

(ii) If $G$ is a bipartite graph, then $\psi(G, \lambda)=\varphi(G, \lambda)$.

Theorem 2.4. Let $G \in \mathcal{G}_{1}$ be a graph with order $n$. Then $\operatorname{det}(2 I+A(G))=n+1$.

Proof. Since $G \in \mathcal{G}_{1}$, then there exists a tree $T$ such that $G=\ell(T)$ and $n(T)=$ $n+1$. Note that $A(G)$ is a matrix of order $n$. Set

$$
\vartheta(\lambda)=|\lambda I-(2 I+A(G))|=\lambda^{n}+a_{1} \lambda^{n}+\cdots+a_{n} .
$$

Suppose that the roots of $\vartheta(\lambda)$ are $\lambda_{1}, \lambda_{2}, \ldots, \lambda_{n}$. Thus,

$$
\operatorname{det}(2 I+A(G))=\lambda_{1} \lambda_{2} \cdots \lambda_{n}
$$

Since $\prod_{i=1}^{n} \lambda_{i}=(-1)^{n} a_{n}$, by (2.1) we have that

$$
\operatorname{det}(2 I+A(G))=(-1)^{n} a_{n} .
$$

Since $T$ is bipartite, from Lemma 2.3 we get that $\psi(T, \lambda)=\varphi(T, \lambda)=\lambda \phi(G, \lambda-2)$. Since $\phi(G, \lambda-2)=|(\lambda-2) I-A(G)|=\vartheta(\lambda)$, we have

$$
\psi(T, \lambda)=\lambda \vartheta(\lambda)=\lambda^{n+1}+a_{1} \lambda^{n}+\cdots+a_{n} \lambda .
$$

Note that $a_{n}=(-1)^{n}(n+1) \tau(T)$, where $\tau(T)$ is the number of spanning trees in $T$ 21]. Consequently, $a_{n}=(-1)^{n}(n+1)$, and therefore, $\operatorname{det}(2 I+A(G))=n+1$.

3. An important graph invariant. Cvetković and Lepović [8] adopted the nomenclature from lattice theory and defined

$$
d_{G}=(-1)^{n} \phi(G,-2)
$$

as the discriminant of an $\mathscr{L}$-graph $G$. Additionally, for an $\mathscr{L}$-graph $G$ they obtained an important graph invariant named star value and showed that its formula is

$$
S=\frac{(-1)^{n}}{(n-k) !} \phi^{(n-k)}(G, \lambda-2)=(-1)^{n} \Pi_{G}(0)=\prod_{i=1}^{k}\left(\lambda_{i}+2\right),
$$

where $\phi^{(p)}(x)$ denotes the $p$-th derivative function of $\phi(x), \Pi_{G}(\lambda)=\prod_{i=1}^{k}\left(\lambda-\left(\lambda_{i}+2\right)\right)$ (it is called the principal polynomial of $G$ [8]) and $\lambda_{1}, \lambda_{2}, \ldots, \lambda_{k}$ are the eigenvalues greater than -2 of $G$. For the discriminant and the star value of $G$, we have the 
following conclusion. If $G$ is an $\mathscr{L}^{0}$-graph, then $d_{G}=0<S$. On the other hand, it is easy to see that $\phi(G, \lambda-2)=\lambda^{n-k} \Pi_{G}(\lambda)$ and then $d_{G}=S$ if $G$ is an $\mathscr{L}^{+}$-graph.

Now we synthesize the above facts into the following definition:

Definition 3.1. Let $G$ be an $\mathscr{L}$-graph of order $n$ and $\Pi_{G}(\lambda)$ the principal polynomial of $G$. Then the star value of $G$ is defined as

$$
\star(G)= \begin{cases}(-1)^{n} \Pi_{G}(0) & \text { if } G \in \mathscr{L}^{0} ; \\ (-1)^{n} \phi(G,-2) & \text { if } G \in \mathscr{L}^{+} .\end{cases}
$$

The following corollary is an immediate consequence of Definition 3.1

Corollary 3.2. Let $G=\cup_{i=1}^{k} G_{i}$ and $H$ be two $\mathscr{L}$-graphs. Then

(i) $\star(G)=\prod_{i=1}^{k} \star\left(G_{i}\right)$;

(ii) if $G$ and $H$ are $A$-cospectral, then $\star(G)=\star(H)$.

Corollary 3.2(ii) indicates that the star value of a graph is a graph invariant determined by the spectrum. The readers will see that it will play an important role in studying the spectral characterization of graphs with least eigenvalue at least -2 .

Note that $\phi(G,-2)=\operatorname{det}(-2 I-A(G))=(-1)^{n} \operatorname{det}(2 I+A(G))$, where $n$ is the order of $G$. Hence, $\operatorname{det}(2 I+A(G))=(-1)^{n} \phi(G,-2)$ and so the following corollary follows from Theorem 2.2 and Definition 3.1

Corollary 3.3. Let $G$ be an $\mathscr{L}^{+}$-graph with order $n$. Then

$$
\star(G)=\left\{\begin{array}{cl}
n+1 & \text { if } G \in \mathcal{G}_{1} ; \\
4 & \text { if } G \in \mathcal{G}_{2} \cup \mathcal{G}_{3} ; \\
3 & \text { if } G \in \mathcal{G}_{4} ; \\
2 & \text { if } G \in \mathcal{G}_{5} ; \\
1 & \text { if } G \in \mathcal{G}_{6} .
\end{array}\right.
$$

For an $\mathscr{L}^{0}$-graph, we pose the following problem.

Problem 3.4. Let $G$ be an $\mathscr{L}^{0}$-graph. Can we determine a formula of its star value $\star(G)$ by means of the parameters of $G$, such as the order, the size, etc?

4. Spectral characterization of line graphs of double stars. The graphs used in this section are shown in Figure 4.1 In this section, we investigate the spectral characterization of line graphs of double stars $S_{a, b}$ and set $b \geq a \geq 0$. Note $\ell\left(S_{0, b}\right)=K_{b+1}$ which has been shown to be a $D A S$-graph (see [11). In what follows we will directly use a well-known fact that if $G$ and $H$ are $A$-cospectral graphs, then they respectively share the same order and size. 

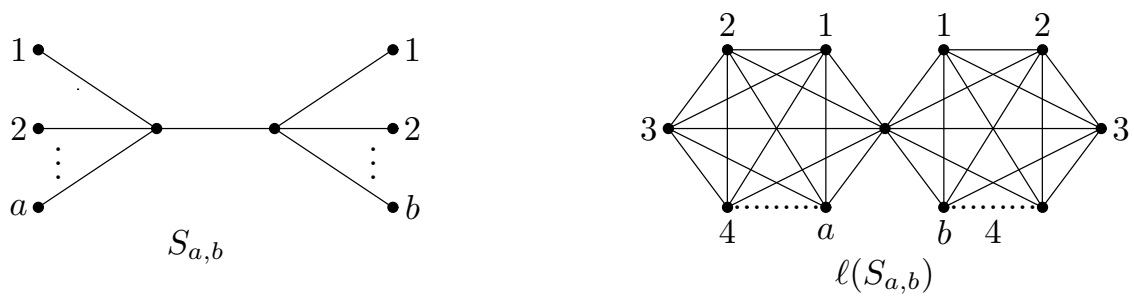

FIG. 4.1. Double star $S_{a, b}$ and its line graph.

Schwenk 25] defined an operation on graphs named generalized composition. If a graph $H$ is labeled and has $s$ vertices, then the graph $G=H\left[H_{1}, H_{2}, \ldots, H_{s}\right]$ is formed by taking the disjoint graphs $H_{1}, H_{2}, \ldots, H_{s}$ and then joining every vertex of $H_{i}$ to every vertex of $H_{j}$ whenever $u_{i}$ is adjacent to $u_{j}$ in $H(1 \leq i, j \leq s)$. Assume that $H_{i}$ is an $r_{i}$-regular graph $(1 \leq i \leq s)$. Then the partition $V(G)=V\left(H_{1}\right) \cup V\left(H_{2}\right) \cup \cdots V\left(H_{s}\right)$ is equitable. Let $T$ be the quotient matrix associated with this partition.

Lemma 4.1. (Schwenk [25]) Let $H_{i}$ be an $r_{i}$-regular graph $(1 \leq i \leq s)$ and let $G=H\left[H_{1}, H_{2}, \ldots, H_{s}\right]$ defined above. Then

$$
\phi(G)=\phi(\lambda I-T) \prod_{i=1}^{s} \frac{\phi\left(H_{i}\right)}{\lambda-r_{i}} .
$$

Lemma 4.2. Let $n\left(\ell\left(S_{a, b}\right)\right)=n$. Then $\rho\left(\ell\left(S_{a, b}\right)\right)>n-2$ if and only if $a \in\{0,1\}$.

Proof. If $b=1$, then $a=0$ or 1 . Thus, $\ell\left(S_{a, b}\right)=P_{2}$ or $P_{3}$ and so the lemma holds. Next set $b \geq 2$. Clearly, $\ell\left(S_{a, b}\right)=P_{3}\left[K_{a}, K_{1}, K_{b}\right]$. Since the partition $V\left(S_{a, b}\right)=$ $V\left(K_{a}\right) \cup V\left(K_{1}\right) \cup V\left(K_{b}\right)$ is equitable, from Lemma 4.1 we get

$$
\phi\left(\ell\left(S_{a, b}\right)\right)=\phi(\lambda I-T) \cdot \frac{\phi\left(K_{a}\right)}{\lambda-(a-1)} \cdot \frac{\phi\left(K_{1}\right)}{\lambda} \cdot \frac{\phi\left(K_{b}\right)}{\lambda-(b-1)},
$$

where $T=\left(\begin{array}{ccc}a-1 & 1 & 0 \\ a & 0 & b \\ 0 & 1 & b-1\end{array}\right)$. A direct calculation shows that

(4.1) $\phi\left(\ell\left(S_{a, b}\right)\right)=(\lambda+1)^{a+b-2}\left[\lambda^{3}-(a+b-2) \lambda^{2}-(2 a-a b+2 b-1) \lambda-(a-2 a b+b)\right]$.

Let $f(\lambda)$ denote the cubic factor. It is easy to see that $\rho\left(\ell\left(S_{a, b}\right)\right)$ is the largest root of $f(\lambda)$. Note that $f(a+b)=a b(a+b+2)>0$ and $f(a+b-1)=-a^{2}-a b-b^{2}+a^{2} b+a b^{2}$. Since $\frac{\partial f(a+b-1)}{\partial a}=(2 a+b)(b-1)>0$, then for $a>2$ we get that

$$
\begin{aligned}
f(a+b-1)>f(2+b-1) & =(b+1)^{2}-5>0 \\
>f(1+b-1) & =-1>f(0+b-1)=-b^{2} .
\end{aligned}
$$


Since $f(\lambda)$ is continuous in the interval $[a+b-1, a+b]$, if $f(\lambda)>0$ for any $\lambda \in$ $[a+b-1, a+b]$, then $f(\lambda)$ has no roots in $[a+b-1, a+b]$. Note that $n-1=a+b$. Since $a+b-1=n-2<\rho\left(\ell\left(S_{a, b}\right)\right)<n-1=a+b$, there exists at least one point $a_{0}+b-1 \in[a+b-1, a+b]$ such that $f\left(a_{0}+b-1\right)<0$. Since $a$ is a positive integer, it follows from (4.2) that $a=1$ or 0 . This establishes the necessity.

Now we show the sufficiency. If $a=0$, then $n=b+1$ and $\ell\left(S_{a, b}\right)=K_{n}$ with $\rho\left(K_{n}\right)=n-1>n-2$. If $a=1$, then $n=b+2$. from (4.1) we get that $\rho\left(S_{1, b}\right)$ is the largest root of

$$
f(\lambda)=\lambda^{3}-(b-1) \lambda^{2}-(b+1) \lambda+(b-1)=0 .
$$

Since $f(n-1)=f(b+1)=b(b+3)>0$ and $f(n-2)=f(b)=-1, n-2<\rho\left(\ell\left(S_{1, b}\right)\right)<$ $n-1$.

Lemma 4.3. Let $b \geq a>1$. Then $\ell\left(S_{a, b}\right)$ has exactly four different eigenvalues $\rho\left(\ell\left(S_{a, b}\right)\right), \lambda_{2}, \lambda_{3}=-1, \lambda\left(\ell\left(S_{a, b}\right)\right)$ such that $\lambda_{2}$ and $\lambda\left(\ell\left(S_{a, b}\right)\right)$ are simple, the multiplicity of -1 is $a+b-2$ and

$$
b+1>\rho\left(\ell\left(S_{a, b}\right)\right)>b>\lambda_{2}>0>\lambda_{3}=-1>\lambda\left(\ell\left(S_{a, b}\right)\right)>-2 .
$$

Proof. We retain the notation of Lemma 4.2, From (4.1) it follows that $\rho\left(\ell\left(S_{a, b}\right)\right)$ and $\lambda\left(\ell\left(S_{a, b}\right)\right)$ are the roots of $f(\lambda)$. Since $f(-1)=a b>0$, then -1 is not a root of $f(\lambda)$. Thus, $\lambda_{2}$ is also a root of $f(\lambda)$ and the multiplicity of -1 is $a+b-2$ by (4.1). A straightforward calculation shows that $f(b+1)=(b-a)(b+4)+4>0, f(b)=-a<$ $0, f(0)=a(b-1)+b(a-1)>0$ and $f(-2)=-a-b-2<0$, and so the inequality (4.3) follows.

Lemma 4.4. No two non-isomorphic line graphs of double stars are A-cospectral.

Proof. Let $\ell\left(S_{a, b}\right)$ and $\ell\left(S_{a^{\prime}, b^{\prime}}\right)$ be $A$-cospectral. Then $a+b=a^{\prime}+b^{\prime}$. From (4.1) it follows that $a b=a^{\prime} b^{\prime}$ (since the constant terms in the cubic factors of $\phi\left(\ell\left(S_{a, b}\right)\right.$ ) and $\phi\left(\ell\left(S_{a^{\prime}, b^{\prime}}\right)\right)$ are equal). Solving the equations $a+b=a^{\prime}+b^{\prime}$ and $a b=a^{\prime} b^{\prime}$ we get that $a=a^{\prime}, b=b^{\prime}$ or $a=b^{\prime}, b=a^{\prime}$.

Theorem 4.5. $\ell\left(S_{1, n-2}\right)$ is a DAS-graph.

Proof. Let $\ell\left(S_{1, n-2}\right)=G$ and let $H$ be any graph $A$-cosptral with $G$. Then $n(H)=n(G)=n$ and $\rho(H)=\rho(G)$. Since $\Delta(H) \geq \rho(H)$, by Lemma 4.2 we get that $\Delta(H)>n-2$ and so $\Delta(H)=n-1$. Hence, $H$ is a connected graph. Since $\lambda(H)=\lambda(G)>-2, H \in \bigcup_{i=1}^{6} \mathcal{G}_{i}$ by Theorem 2.1. From Corollary 3.3 it follows that $\star(H)=\star(G)=n+1$. If $n=2$ or 3 , then $G=P_{2}$ or $P_{3}$ which has been proved to be a DAS-graph. Now set $n \geq 4$; then $\star(H) \geq 5$, and therefore, $H \in \mathcal{G}_{1}$ by Corollary 3.3. Thus, $H=\ell(T)$, where $T$ is a tree. Since $e_{1}$ has degree $n-1$ in $H$, the other 
edges of $T$ must be adjacent to $e_{1}$, and this implies that $T$ is a double star $S_{a, b}$. From Lemma 4.4 it follows that $H \cong G$.

Lemma 4.6. 26] The double star $S_{a, b}$ is determined by the Laplacian spectrum.

LEMMA 4.7. [27] A graph has exactly one positive eigenvalue if and only if its non-isolated vertices form a complete multipartite graph.

Theorem 4.8. Let $n=a+b+1$ and $b \geq a \geq 2$. Then $\ell\left(S_{a, b}\right)$ is a DAS-graph.

Proof. Let $\ell\left(S_{a, b}\right)=G$ and let $H$ be any graph $A$-cospectral with $G$. Then $n(H)=n(G)=n$. We first show the following consequence:

Claim: $H$ is a connected graph.

Assume by way of contradiction that $H=\bigcup_{i=1}^{k} H_{i}$, where $k>1$ and $H_{i}(1 \leq$ $i \leq k)$ is a connected component of $H$. Since $\lambda(H)=\min \left\{\lambda\left(H_{i}\right) \mid i=1, \ldots, k\right\}=$ $\lambda(G)>-2$, by Theorem 2.1 we get that $H_{i} \in \cup_{j=1}^{6} \mathcal{G}_{j}(i=1, \ldots, k)$. Without loss of generality, set $\lambda(H)=\lambda\left(H_{1}\right)=\lambda\left(\ell\left(S_{a, b}\right)\right)$. Note that $\ell\left(S_{a, b}\right)$ has only one eigenvalue $\lambda\left(\ell\left(S_{a, b}\right)\right)$ less than -1 . Hence, $\lambda\left(H_{1}\right)<-1$ and $\lambda\left(H_{i}\right) \geq-1(i=2, \ldots, k)$. Since $H$ has no eigenvalue 0 , then each $H_{i}$ is not an isolated vertex, and therefore, $H_{i}$ has at least one edge $(2 \leq i \leq k)$. Consequently, $\lambda\left(H_{i}\right) \leq-1$ and so $\lambda\left(H_{i}\right)=-1$ which implies that $H_{i}$ is a complete graph $K_{a_{i}}(2 \leq i \leq k)$. Thus,

$$
H=H_{1} \cup K_{a_{2}} \cup \cdots \cup K_{a_{k}} \text {, where } a_{2} \geq a_{3} \geq \cdots \geq a_{k} .
$$

From (4.3) it follows that $\rho(H)=\rho\left(\ell\left(S_{a, b}\right)\right)$ is not an integer, which, together with $\rho(H)=\max \left\{\rho\left(H_{1}\right), \rho\left(K_{a_{2}}\right)=a_{2}-1\right\}$, leads to $\rho(H)=\rho\left(H_{1}\right)$.

If $H_{1}$ has exactly two different eigenvalues $\rho\left(H_{1}\right)$ and $\lambda\left(H_{1}\right)$, then $H_{1}$ must be a complete graph whose spectral radius $\rho\left(H_{1}\right)=n\left(H_{1}\right)-1$ is an integer, a contradiction. Hence, $H_{1}$ has at least three different eigenvalues $\rho\left(H_{1}\right), \lambda_{2}\left(H_{1}\right), \lambda\left(H_{1}\right)$.

Note that $\operatorname{Spec}\left(K_{a_{i}}\right)=\left\{a_{i}-1,-1^{a_{i}-1}\right\}(i=2, \ldots, k)$. If $a_{i} \neq a_{j}(2 \leq i \neq j \leq k)$, then $H$ has at least three positive eigenvalues $\rho(H), a_{i}-1, a_{j}-1$ which contradicts the fact that $H$ has exactly two positive eigenvalues (see (4.3)). Thus, $a_{2}=\cdots=a_{k}$ which shows that the multiplicity of eigenvalue $a_{2}-1$ is $k-1$.

Since the second largest positive eigenvalue of $H$ is simple (see Lemma 4.3), then $k-1=1$ and so $k=2$ and $H=H_{1} \cup K_{a_{2}}$. Note again that $H$ has four different eigenvalues $\rho(H)=\rho\left(H_{1}\right)=\rho\left(\ell\left(S_{a, b}\right)\right), \lambda_{2}(H)=\lambda_{2}\left(\ell\left(S_{a, b}\right)\right), \lambda_{3}(H)=$ $\lambda_{3}\left(\ell\left(S_{a, b}\right)\right)=-1, \lambda(H)=\lambda\left(H_{1}\right)=\lambda\left(\ell\left(S_{a, b}\right)\right)$. If $H_{1}$ has four different eigenvalues $\rho\left(H_{1}\right), \lambda_{2}\left(H_{1}\right), \lambda_{3}\left(H_{1}\right), \lambda\left(H_{1}\right)$, then $\lambda_{2}(H)=\lambda_{2}\left(H_{1}\right)=a_{2}-1$ and $\lambda_{3}(H)=\lambda_{3}\left(H_{1}\right)=$ -1 which contradicts the fact that $\lambda_{2}(H)$ is simple. So, $H_{1}$ has exactly three different eigenvalues $\rho\left(H_{1}\right), \lambda_{2}\left(H_{1}\right), \lambda\left(H_{1}\right)$ and the possibly different eigenvalues of $H$ lie in $\left\{\rho\left(H_{1}\right), \lambda_{2}\left(H_{1}\right), \lambda\left(H_{1}\right)\right\} \cup\left\{a_{2}-1,-1\right\}$. 
If $\lambda_{2}\left(H_{1}\right)>0$, then $\lambda_{2}\left(H_{1}\right)=a_{2}-1$ which contradicts that the second largest eigenvalue of $H$ is simple. If $\lambda_{2}\left(H_{1}\right)<0$, then $\lambda_{2}\left(H_{1}\right)=-1$ and thus $H_{1}$ has only one positive eigenvalue. By Lemma 4.7 we get that $H_{1}$ is a complete multipartite graph $K_{c_{1}, c_{2}, \ldots, c_{s}}$. It has been proved that $H_{1}=K_{c_{1}, c_{2}, \ldots, c_{s}}$ has eigenvalue 0 with multiplicity $n\left(H_{1}\right)-s$ (see [5] pp. 74). Since $H$ has no eigenvalue 0 , then $n\left(H_{1}\right)=s$ and so $H_{1}$ is a complete graph $K_{n\left(H_{1}\right)}$ with integral spectral radius $n\left(H_{1}\right)-1$, a contradiction. This finishes the proof of the Claim.

From Corollaries 3.2 and 3.3 we get that $\star(H)=\star(G)=n+1$. By the condition of the theorem, we have $\star(H) \geq 6$ and thus $H \in \mathcal{G}_{1}$ by Theorem [2.1. So, there exists a tree $T$ such that $H=\ell(T)$. From $\phi\left(\ell\left(S_{a, b}\right), \lambda\right)=\phi(\ell(T), \lambda)$ it follows that $\lambda \phi\left(\ell\left(S_{a, b}\right), \lambda-2\right)=\lambda \phi(\ell(T), \lambda-2)$ which by Lemma 2.3(i) yields $\varphi(T, \lambda)=\varphi\left(S_{a, b}, \lambda\right)$ and so $\psi(T, \lambda)=\psi\left(S_{a, b}, \lambda\right)$ by Lemma 2.3(ii). From Lemma 4.6] it follows that $T \cong S_{a, b}$ and so $H=\ell(T) \cong \ell\left(S_{a, b}\right)$.

Combining the fact that $\ell\left(S_{0, b}\right)=K_{b+1}$ is a DAS-graph and Theorems 4.5 and 4.8 we immediately obtain the main result of this section:

Theorem 4.9. Let $b \geq a \geq 0$. Then $\ell\left(S_{a, b}\right)$ is a DAS-graph.

Acknowledgment. The authors are grateful to the anonymous referee for his or her careful reading and observations which led to an improved presentation of this paper.

\section{REFERENCES}

[1] R. Boulet. Disjoint unions of complete graphs characterized by their Laplacian spectrum. Electron. J. Linear Algebra, 18:773-783, 2009.

[2] A.E. Brouwer, A.M. Cohen, and A. Neumaier. Distance-Regular Graphs. Springer-Verlag, Berlin, 1989.

[3] P.J. Cameron, J.M. Goethal, J.J. Seidel, and E.E. Shult. Line graphs, root systems, and elliptic geometry. J. Algebra, 43:305-327, 1976.

[4] P.J. Cameron and J.H. van Lint. Designs, Graphs, Codes and Their Links. Cambridge University Press, Cambridge, 1991.

[5] D. Cvetković, M. Doob, and H. Sachs. Spectra of Graphs - Theory and Applications, III. Johan Ambrosius Bart Verlag, Heidelberg-Leipzig, 1995.

[6] D. Cvetković and M. Lepović. A table of cospectral graphs with least eigenvalue at least -2 . Availiable at http://www.mi.sanu.ac.yu/projects/results1389.htm.

[7] D. Cvetković and M. Lepović. Sets of cospectral graphs with least eigenvalue at least 2 and some related results. Bull. Acad. Serbe Sci. Arts Cl. Sci. Math. Natur., 129:85-102, 2004.

[8] D. Cvetković and M. Lepović. Cospectral graphs with least eigenvalue at least -2 . Publ. Inst. Math., Nouv. Sér., 78:51-63, 2005.

[9] D. Cvetković, P. Rowlinson, and S.K. Simić. Spectral Generalizations of Line Graphs, On Graphs with Least Eigenvalue -2. Cambridge University Press, Cambridge, 2004.

[10] D.M. Cvetković, P. Rowlinson, and S.K. Simić. Signless Laplacian of finite graphs. Linear Algebra Appl., 423:155-171, 2007. 
[11] E.R. van Dam and W.H. Haemers. Which graphs are determined by their spectrum? Linear Algebra Appl., 373:241-272, 2003.

[12] E.R. van Dam and W.H. Haemers. Developments on spectral characterizations of graphs. Discrete Math., 309:576-586, 2009.

[13] M. Doob and D. Cvetković. On spectral characterizations and embeddings of graphs. Linear Algebra Appl., 27:17-26, 1979.

[14] C.D. Godsil and B.D. McKay. Constructing cospectral graphs. Aequationes Math., 25:257-268, 1982.

[15] C. Godsil and G. Royle. Algebraic Graph Theory. Springer-Verlag, New York, 2001.

[16] H.H. Günthard and H.Primas. Zusammenhang von Graphentheorie und MO-Theorie von Molekeln mit Systemen konjugierter Bindungen. Helvetica Chimica Acta, 39:1645-1653, 1956.

[17] W.H. Haemers and E. Spence. Enumeration of cospectral graphs. European J. Combin., 25:199$211,2004$.

[18] F. Harary, C. King, A. Mowshowitz, and R. Read. Cospectral graphs and digraphs. Bull. London Math. Soc., 3:321-328, 1971.

[19] A.J. Hoffman. $-1-\sqrt{2}$ ? Combinatorial Structures and their Applications. Gordon \& Branch, New York, 173-176, 1970.

[20] C.R. Johnson and M. Newman. A note on cospectral graphs. J. Combin. Theory. Ser. B, 28:96-103, 1980.

[21] A.K. Kelmans and V.M. Chelnokov. A certain polynomial of a graph and graphs with an extremal number of trees. J. Combin. Theory Ser. B, 16:197-214, 1974.

[22] M.H. Liu, B.L. Liu, and F.Y. Wei, Graphs determined by their (signless) Laplacian spectra. Electron. J. Linear Algebra, 22:112-124, 2011.

[23] M. Mirzakhah and D. Kiani. The sun graph is determined by its signless Laplacian spectrum. Electron. J. Linear Algebra, 20:610-620, 2010.

[24] G.R. Omidi and E. Vatandoost. Starlike trees with maximum degree 4 are determined by their signless Laplacian spectra. Electron. J. Linear Algebra, 20:274-290, 2010.

[25] A.J. Schwenk. Computing the characteristic polynomial of a graph. In R. Bari and F. Harary (editors) Graphs and Combinatorics, Lecture Notes in Mathematics, Springer-Verlag, Berlin, 406:153-172, 1974.

[26] X.L. Shen and Y.P. Hou. Some trees determined by their Laplacian spectra (in Chinese). J. Nat. Sci. Hunan Norm. Univ., 29:21-24, 2006.

[27] J.H. Smith. Some properties of the spectrum of a graph. Combinatorial Structures and their Applications, Gordon and Breach, New York, 403-406, 1970.

[28] J.F. Wang, F. Belardo, Q.X. Huang, and B. Borovicanin. On the two largest $Q$-eigenvalues of graphs. Discrete Math., 310:2858-2866, 2010.

[29] J.F. Wang, Q.X. Huang, F. Belardo, and E.M. Li Marzi. Spectral characterizations of dumbbell graphs. Electron. J. Combin., 17:21-16, \#R42, 2010.

[30] J.F. Wang, Q.X. Huang, F. Belardo, and E.M. Li Marzi. On the spectral characterizations of $\infty$-graphs. Discrete Math., 310:1845-1855, 2010.

[31] J.F. Wang, S.K. Simić, Q.X. Huang, F. Belardo, and E.M. Li Marzi. Laplacian spectral characterization of disjoint union of paths and cycles. Linear Multilinear Algebra, 59:531-539, 2011. 